\title{
ABUNDANCES OF HEAVY ELEMENTS IN HALO STARS
}

\author{
G. ZHAO \\ Beijing Astronomical Observatory \\ Chinese Academy of Sciences \\ Beijing 100080, China
}

We reanalysed the observational material of high resolution, high signalto-noise spectra for a sample of 27 halo stars. The data were collected with the Coudé Echelle Spectrometer (CES) fed by the $1.4 \mathrm{~m}$ Coudé Auxiliary Telescope (CAT) at the European Southern Observatory (La Silla, Chile). Data reduction are the same as described in a previous paper (Zhao and Magain, 1991). However, the non-linearity present in the data was corrected by using an empirical fitting formula (Gosset and Magain, 1993). The atmospheric parameters of these program stars have been determined. Effective temperatures were derived from $b-y$ and $V-K$ color indices by using the calibration of Magain. Surface gravities were determined by forcing the FeII lines to indicate the same abundance as the high excitation FeI lines. Metallicities were derived from Strömgren $m_{1}$ indices. The abundances of a number of heavy elements, namely, strontium, yttrium, zirconium, barium, lanthanum, cerium and europium, are deduced on the basis of a new data reduction. The main results are: (1) The elements of the first peak, strontium-yttrium-zirconium, show the odd-even effect: strontium and zirconium are less deficient than yttrium in halo stars. (2) The yttrium abundance is constant for all stars with $[\mathrm{Fe} / \mathrm{H}]>-2.5$. (3) $[\mathrm{Ba} / \mathrm{Fe}]$ values are 0.25 dex higher than solar for $[\mathrm{Fe} / \mathrm{H}]>-1.8$. (4) The barium overdeficiency increases with decreasing metallicity when $[\mathrm{Fe} / \mathrm{H}]<$ -1.8. (5) Lanthanum and cerium are overdeficient with respect to iron. (6) Europium is overabundant for $-1.0>[\mathrm{Fe} / \mathrm{H}]>-2.0$.

\section{References}

Gosset, E., Magain, P. (1993) On the linearity of ESO CCD $\sharp 9$ at CAT+CES, ESO Messenger, 73, pp. 13-17

Zhao, G., Magain, P. (1991) Abundances of neutron capture elements in metal-poor dwarfs, $A \& A, 244$, pp. 425-432 